\title{
ARTIGO DE REVISÃO Síndromes neuropsiquiátricas associadas a acidentes vasculares encefálicos: revisão de literatura
}

\author{
Neuropsychiatric syndromes associated with stroke: review of the literature \\ Vinicius Sousa Pietra Pedroso', Leonardo Cruz de Souza', Antônio Lúcio Teixeira'
}

\author{
Palavras-chave \\ Acidente vascular \\ encefálico, doença \\ cerebrovascular, \\ neuropsiquiatria, depressão, \\ ansiedade, demência.
}

\section{Keywords}

Stroke, cerebrovascular disease, neuropsychiatry, depression, anxiety, dementia.

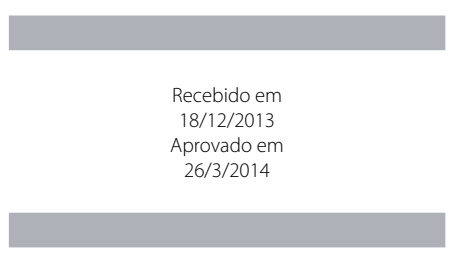

DOI: 10.1590/0047-2085000000021

\section{RESUMO}

Objetivo: Revisar as principais síndromes neuropsiquiátricas associadas ao acidente vascular encefálico (AVE), suas características clínicas, impacto sobre a recuperação dos pacientes, tratamento, suas possíveis relações com a fisiopatologia dos AVE e, quando possível, contextualizá-las à realidade brasileira. Métodos: Foram realizadas buscas nas bases de dados PubMed/ MedLine e SciELO/Lilacs com os termos "stroke" e "cerebrovascular disease" em combinações com "neuropsychiatry", "neuropsychiatric disorders", "psychiatry", "psychiatric disorders", "depression", "anxiety" e "dementia", com ênfase nos últimos dez anos. Resultados: Foram revisadas as síndromes neuropsiquiátricas pós-AVE, incluindo depressão, ansiedade, transtorno da expressão emocional involuntária, labilidade emocional, irritabilidade, raiva, reação catastrófica, apatia, demência, mania e psicose, de acordo com os objetivos propostos. Conclusão: É notória a escassez de informações sobre o manejo terapêutico das complicações neuropsiquiátricas secundárias aos $A V E$, especialmente diante do impacto em saúde pública representado pelas doenças cerebrovasculares. Com a evolução da abordagem precoce a esses pacientes e o consequente aumento de sua sobrevida, o aprofundamento do conhecimento sobre o desenvolvimento e o tratamento dos transtornos neuropsiquiátricos parece ter maior potencial para melhorar o desfecho e a qualidade de vida dos indivíduos que sofreram AVE.

\begin{abstract}
Objective: To review the main neuropsychiatric syndromes associated with stroke, their clinical features, impact over functional recovery, therapeutics, putative relations to stroke pathophysiology and, when possible, to contextualize them to the Brazilian reality. Methods: It was performed a search on PubMed/MedLine and SciELO/Lilacs databases, using the terms "stroke" and "cerebrovascular disease" combined with "neuropsychiatry", "neuropsychiatric disorders", "psychiatry", "psychiatric disorders", "depression", "anxiety" and "dementia". Results: According to the proposed objectives, we reviewed the stroke-related syndromes characterized by depression, anxiety, emotional lability, irritability, anger, catastrophic reaction, apathy, dementia, mania and psychosis. Conclusion: These findings emphasize the lack of information on the therapeutic management of neuropsychiatric complications secondary to stroke, especially considering the burden on public health represented by cerebrovascular diseases. Following the improvement in the survival rates with the early strategies to stroke, the advancement of knowledge on neuropsychiatric disorders seem to have the greatest potential to improve the quality of life of patients affected by stroke.
\end{abstract}

1 Universidade Federal de Minas Gerais (UFMG), Faculdade de Medicina, Laboratório Interdisciplinar de Investigação Médica. UFMG, Hospital das Clínicas, Serviço de Neurologia, Grupo de Neuropsiquiatria.

Endereço para correspondência: Vinicius Sousa Pedroso e Antônio Lúcio Teixeira

Laboratório Interdisciplinar de Investigação Médica (LIIM), sala 281, Faculdade de Medicina, UFMG.

Av. Alfredo Balena, 190, Santa Efigênia

30130-100 - Belo Horizonte, MG, Brasil

E-mail: viniciuspietra@yahoo.com.br 


\section{INTRODUÇÃO}

O desenvolvimento rápido de alterações neurológicas focais ou globais que duram mais de 24 horas ou levam ao óbito, excluindo-se causas não vasculares, corresponde à definição de acidente vascular encefálico (AVE), de acordo com a Organização Mundial da Saúde!.

Embora nos últimos 50 anos a incidência de AVE e a mortalidade por causa dele tenham sido reduzidas nos países desenvolvidos, especialmente devido ao avanço na abordagem da isquemia cerebral aguda e à implementação de estratégias para controle de fatores de risco, como hipertensão arterial sistêmica (HAS) e diabetes, os AVE ainda representam um grande problema de saúde pública, sendo a terceira causa de morte no mundo ocidental e a principal causa de incapacidade permanente ${ }^{2,3}$.

No Brasil, os dados epidemiológicos disponíveis vêm de poucos estudos desenvolvidos em cidades isoladas e não permitem generalização nacional, especialmente considerando-se as disparidades regionais ${ }^{4}$. No entanto, as informações existentes permitem afirmar que os AVE constituem a principal causa de morte no país e, se há uma tendência de redução da mortalidade similar àquela observada nos países desenvolvidos, esta reflete a realidade das regiões Sul e Sudeste ${ }^{5}$.

Entre os indivíduos sobreviventes, cerca de dois terços apresentam algum grau de incapacidade permanente que requer cuidados de reabilitação, e a recorrência de eventos isquêmicos contribui ainda mais para o aumento da morbidade $^{6}$. Acima dos 60 anos de idade, os AVE se destacam como a condição que determina a adoção dos procedimentos mais dispendiosos para o sistema público de saúde e com a maior taxa de óbitos ${ }^{6,7}$.

Além das sequelas neurológicas, como déficits motores e sensitivos, as alterações neuropsiquiátricas têm sido reconhecidas como determinantes da recuperação ${ }^{7}$. Há muito se observou que indivíduos acometidos por AVE apresentam maior incidência de transtornos de humor e da cognição em comparação àqueles com outras doenças crônicas ${ }^{8}$. No entanto, nos últimos 20 anos, outras síndromes neuropsiquiátricas, como transtorno de ansiedade generalizada (TAG), apatia, transtornos da expressão emocional, psicose, mania secundária, alterações da personalidade, entre outras, têm sido descritas após AVE. Além de impactar a recuperação neurológica, exercem significativa influência na vida profissional e nas relações interpessoais dos pacientes, de seus familiares e de seus cuidadores, modificando sua autonomia, sua autoestima e sua qualidade de vida (QV) em geral ${ }^{9}$.

O presente trabalho tem como objetivo oferecer uma revisão narrativa sobre as principais síndromes neuropsiquiátricas associadas ao AVE, suas características clínicas, impacto sobre a recuperação dos pacientes e possível fisiopatologia.

\section{MÉTODOS}

Este trabalho é uma revisão narrativa da literatura que teve como fonte de pesquisa as bases de dados PubMed/ MedLine e SciELO/Lilacs. Os termos de busca foram "stroke" e "cerebrovascular disease" em combinações com "neuropsychiatry", "neuropsychiatric disorders", "psychiatry", "psychiatric disorders", "depression", "anxiety" e "dementia", em todos os campos. O período dos últimos dez anos foi utilizado como filtro inicial de busca eletrônica. Foram avaliados artigos nos idiomas português, inglês, espanhol e francês. Consultas às referências dos artigos selecionados, bem como a livros-texto, foram utilizadas como estratégia de busca adicional. Todos os autores participaram da elaboração do texto. Foram selecionados, a critério dos autores e com base na força de evidência e originalidade dos trabalhos, artigos de revisão, revisões sistemáticas, metanálises, artigos originais e relatos de casos para a caracterização de aspectos gerais das síndromes neuropsiquiátricas associadas aos AVE e de questões próprias de cada síndrome em particular, com foco especial em trabalhos realizados no Brasil, de forma a salientar a realidade nacional.

\section{RESULTADOS}

A busca no PubMed/MedLine resultou em 11.251 trabalhos, sendo 5.755 publicados nos últimos dez anos e 2.268 artigos de revisão. Já na base de dados SciELO/Lilacs, a busca resultou em 241 trabalhos. Procurou-se priorizar estudos com maior tamanho amostral ou maior nível de evidência, a partir dos quais se definiram os temas a serem abordados na revisão.

\section{Síndromes neuropsiquiátricas pós-AVE: visão geral}

Inúmeros autores procuraram determinar a frequência de manifestações neuropsiquiátricas em pacientes com AVE, em momentos diferentes após o evento isquêmico. O quadro 1 apresenta uma distribuição geral de síndromes neuropsiquiátricas em pacientes afetados por AVE de acordo com a literatura.

Angelelli et al. ${ }^{10}$ procuraram identificar a prevalência de sintomas neuropsiquiátricos entre pacientes com AVE. Observaram uma divisão entre manifestações típicas da fase aguda e da fase crônica, encontrando o ponto de mudança em torno do sexto mês pós-AVE. O perfil "crônico" manteve-se estável por, ao menos, um ano. Assim, na fase aguda, as alterações mais comuns foram agitação, euforia e comportamento motor aberrante. Já na fase crônica, predominaram apatia e transtornos relacionados ao sono, como insônia, hipersonia ou outros comportamentos inadequados, como levantar-se e perambular pela casa à noite. Os sintomas de depressão e de irritabilidade foram frequentes em ambas as fases. 
Quadro 1. Características dos estudos que analisaram as principais síndromes neuropsiquiátricas associadas ao AVE

\begin{tabular}{|c|c|c|c|c|c|}
\hline Síndromes e sintomas & Autor (ano) & $\mathrm{n}$ & Contexto & Tempo pós-AVE & Prevalência (\%) \\
\hline \multirow[t]{15}{*}{ Depressão } & Robinson et al. (1983) & 103 & $\mathrm{H}$ & 2 semanas & 47 \\
\hline & Robinson et al. (1984) & 61 & A & $\begin{array}{l}3 \text { meses } \\
6 \text { meses }\end{array}$ & $\begin{array}{l}49 \\
60\end{array}$ \\
\hline & Robinson et al. (1987) & 65 & A & $\begin{array}{l}12 \text { meses } \\
24 \text { meses }\end{array}$ & $\begin{array}{l}33 \\
42\end{array}$ \\
\hline & Eastwood et al. (1989) ${ }^{14}$ & 87 & UR & $1-3$ meses & 50 \\
\hline & Morris et al. $(1990)^{15}$ & 99 & UR/A & $\begin{array}{l}2 \text { meses } \\
15 \text { meses }\end{array}$ & $\begin{array}{l}32 \\
12\end{array}$ \\
\hline & House et al. $(1991)^{16}$ & 89 & c & Primeiros 12 meses & 23 \\
\hline & Astrom et al. $(1993)^{17}$ & 80 & $H / A$ & $\begin{array}{l}0-3 \text { meses } \\
12 \text { meses } \\
24 \text { meses } \\
36 \text { meses }\end{array}$ & $\begin{array}{l}31 \\
16 \\
19 \\
29\end{array}$ \\
\hline & Burvill et al. $(1995)^{18}$ & 294 & c & 4 meses & 23 \\
\hline & $\mathrm{Ng}$ et al. $(1995)^{19}$ & 52 & UR & Primeiro mês & 55 \\
\hline & Kotila et al. $(1998)^{20}$ & 321 & A & $\begin{array}{l}3 \text { meses } \\
12 \text { meses }\end{array}$ & $\begin{array}{l}41-54 \\
42-55\end{array}$ \\
\hline & Angelelli et al. $(2004)^{10}$ & 124 & $H / A$ & 2-12 meses & 61,3 \\
\hline & Hackett et al. $(2005)^{21}$ & 51 estudos & Metanálise & $0-24$ meses & 33 \\
\hline & Buijck et al. $(2012)^{22}$ & 145 & IEQ & - & 33 \\
\hline & Ayerbe et al. $(2013)^{23}$ & 4022 & c & Primeiros 15 anos & $29-39$ \\
\hline & Ayerbe et al. $(2013)^{24}$ & 50 estudos & Metanálise & $0-5$ anos & 29 \\
\hline \multirow[t]{3}{*}{ Irritabilidade e raiva } & Angelelli et al. $(2004)^{10}$ & 124 & $H / A$ & 2-12 meses & 33 \\
\hline & Skånér et al. $(2007)^{25}$ & 187 & C & $\begin{array}{l}3 \text { meses } \\
12 \text { meses }\end{array}$ & $\begin{array}{l}46 \\
34\end{array}$ \\
\hline & Buijck et al. $(2012)^{22}$ & 145 & IEQ & - & 12 \\
\hline \multirow{3}{*}{$\begin{array}{l}\text { Transtornos alimentares e do } \\
\text { apetite }\end{array}$} & Angelelli et al. $(2004)^{10}$ & 124 & $H / A$ & 2-12 meses & 33 \\
\hline & Skånér et al. (2007) & 187 & c & $\begin{array}{l}3 \text { meses } \\
12 \text { meses }\end{array}$ & $\begin{array}{l}34 \\
21\end{array}$ \\
\hline & Buijck et al. $(2012)^{22}$ & 145 & IEQ & - & 19 \\
\hline \multirow[t]{2}{*}{ Agitação } & Angelelli et al. $(2004)^{10}$ & 124 & $\mathrm{H} / \mathrm{A}$ & 2-12 meses & 28,2 \\
\hline & Buijck et al. $(2012)^{22}$ & 145 & IEQ & - & 5 \\
\hline \multirow[t]{4}{*}{ Apatia } & Starkstein et al. $(1993)^{26}$ & 80 & $H$ & Primeiras 2 semanas & 11 \\
\hline & Angelelli et al. $(2004)^{10}$ & 124 & $\mathrm{H} / \mathrm{A}$ & 2-12 meses & 26,6 \\
\hline & Brodaty et al. $(2005)^{27}$ & 167 & A & $3-6$ meses & 26,7 \\
\hline & Buijck et al. $(2012)^{22}$ & 145 & IEQ & - & 9 \\
\hline \multirow[t]{3}{*}{ Ansiedade } & Angelelli et al. $(2004)^{10}$ & 124 & $H / A$ & 2-12 meses & 26,6 \\
\hline & Buijck et al. $(2012)^{22}$ & 145 & IEQ & - & 15 \\
\hline & Burton et al. $(2013)^{28}$ & 44 estudos & Metanálise & $\begin{array}{l}\text { Primeiro mês } \\
1-5 \text { meses } \\
\text { Após sexto mês }\end{array}$ & $\begin{array}{l}20 \\
23 \\
24\end{array}$ \\
\hline \multirow[t]{3}{*}{ Distúrbios noturnos } & Angelelli et al. $(2004)^{10}$ & 124 & $H / A$ & 2-12 meses & 17,7 \\
\hline & Skånér et al. $(2007)^{25}$ & 187 & c & $\begin{array}{l}3 \text { meses } \\
12 \text { meses }\end{array}$ & $\begin{array}{l}41 \\
31\end{array}$ \\
\hline & Buijck et al. $(2012)^{22}$ & 145 & IEQ & - & 18 \\
\hline TEEI & $\begin{array}{l}\text { Calvert et al. }(1998)^{29} \\
\text { Piamarta et al. }(2004)^{30}\end{array}$ & $\begin{array}{l}448 \\
33\end{array}$ & $\begin{array}{l}\text { A } \\
\mathrm{H}\end{array}$ & $\begin{array}{c}1 \text { mês } \\
2 \text { semanas }\end{array}$ & $\begin{array}{l}21,5 \\
48,5\end{array}$ \\
\hline \multirow{2}{*}{$\begin{array}{l}\text { Comportamento motor } \\
\text { aberrante }\end{array}$} & Angelelli et al. $(2004)^{10}$ & 124 & $H / A$ & 2-12 meses & 10,5 \\
\hline & Buijck et al. $(2012)^{22}$ & 145 & IEQ & - & 1 \\
\hline \multirow[t]{2}{*}{ Desinibição } & Angelelli et al. $(2004)^{10}$ & 124 & $H / A$ & 2-12 meses & 9,7 \\
\hline & Buijck et al. $(2012)^{22}$ & 145 & IEQ & - & 1 \\
\hline \multirow[t]{2}{*}{ Delírios } & Angelelli et al. $(2004)^{10}$ & 124 & $H / A$ & 2-12 meses & 1,6 \\
\hline & Buijck et al. $(2012)^{22}$ & 145 & IEQ & - & 3 \\
\hline \multirow[t]{2}{*}{ Alucinações } & Angelelli et al. $(2004)^{10}$ & 124 & $H / A$ & 2-12 meses & 0,8 \\
\hline & Buijck et al. $(2012)^{22}$ & 145 & IEQ & - & 4 \\
\hline
\end{tabular}

A: ambulatorial; C: comunidade; H: hospitalar; IEQ: instalação de enfermagem qualificada; TEEl: transtorno da expressão emocional involuntária; UR: unidade de reabilitação. 


\section{Depressão pós-AVE}

A investigação sistemática das síndromes depressivas secundárias aos AVE foi desenvolvida principalmente pelo grupo do professor Robert Robinson, da Universidade de lowa, Estados Unidos ${ }^{31}$. Desde então, foram relatadas prevalências que variam de $18 \%$ a $60 \%$, estabelecendo a depressão (maior e menor) como o transtorno neuropsiquiátrico mais comum após AVE. A variabilidade na prevalência deve-se aos diferentes questionários e critérios utilizados para definição diagnóstica, bem como às diferenças entre as populações estudadas e o tempo decorrido após o AVE. De acordo com o DSM-V (Diagnostic and Statistical Manual of Mental Disorders, fourth edition), o transtorno depressivo maior corresponde à presença de, ao menos, quatro sintomas acessórios, além de humor deprimido ou anedonia. Já o episódio depressivo menor corresponde a um diagnóstico de pesquisa do DMS-IV, caracterizado pela presença de dois a cinco sintomas depressivos, incluindo humor deprimido ou anedonia. No novo DSM-V, esse construto foi removido dos diagnósticos de pesquisa e aparentemente incorporado à categoria Outros Transtornos Depressivos Especificados: Episódio Depressivo com Sintomas Insuficientes.

A prevalência média de depressão pós-AVE (DPA) entre pacientes hospitalizados em fase aguda gira em torno de 22\% para depressão maior e 17\% para depressão menor. Em amostras ambulatoriais, é de 23\% para depressão maior e de 35\% para depressão menor, enquanto amostras comunitárias exibem prevalências médias de 13\% e 10\%, respectivamente ${ }^{8}$. Uma recente metanálise observou que a prevalência de depressão em qualquer momento após AVE era de 29\%32.

Uma coorte de 4.022 pacientes acompanhados por 15 anos evidenciou a história dinâmica da DPA ${ }^{33}$. Segundo Robinson et al. ${ }^{13}$, episódios depressivos maiores frequentemente se atenuam, sem remissão completa, um ano após o evento. Na coorte, mais da metade dos pacientes apresentou depressão, com prevalência estável em torno de 30\% durante o acompanhamento ${ }^{33}$. A maior parte dos indivíduos apresentou remissão um ano após o AVE, exibindo episódios depressivos de duração relativamente curta. No entanto, o risco de recorrência desses episódios em longo prazo foi elevado, corroborando a relação entre episódios curtos e prevalência estável.

No Brasil, há poucos dados sobre a epidemiologia da DPA. Em Fortaleza, avaliando-se a QV de indivíduos em torno de dois a seis anos pós-AVE, observou-se prevalência de $40 \%$ de sintomas depressivos, predominando leves a moderados $^{32}$. A presença dos sintomas depressivos foi o fator mais importante na redução da QV. Similarmente, Souza et al. avaliaram pacientes com doença de Chagas e AVE, observando que a QV era mais influenciada pelos sintomas depressivos do que pela incapacidade neurológica ${ }^{33}$. Por sua vez, Carod-Artal et al. também demonstraram que depressão, incapacidade e déficit motor eram os principais determinantes da QV relacionada à saúde em pacientes com AVE, sendo depressão o mais forte preditor de redução da QV, especialmente entre mulheres ${ }^{34}$. O quadro 2 reúne trabalhos brasileiros que registraram a frequência de DPA.

Estudos longitudinais sugerem que depressões maior e menor são determinantes de incapacidade, fracasso no retorno ao trabalho, prejuízo no funcionamento interpessoal e mortalidade ${ }^{39}$. A relação entre DPA e comprometimento funcional é complexa. Pacientes afetados por AVE frequentemente sofrem algum grau de incapacidade em longo prazo. Embora a maioria dos indivíduos afetados experimente algum grau de recuperação neurológica, com melhora do desempenho de atividades de vida diária (AVD), tem-se observado que pacientes com DPA apresentam significativamente maior incapacidade para AVD do que pacientes eutímicos e com déficits neurológicos comparáveis ${ }^{10}$. Essas observações sugerem um fenômeno de reciprocidade, no qual a depressão influencia a recuperação das AVD e o comprometimento das AVD influencia a gravidade e a duração de depressão.

Os critérios do DSM-V para diagnóstico de DPA correspondem àqueles para transtorno depressivo devido a uma condição médica, que deve ser diferenciado da desmoralização pós-AVE. Diversos autores buscaram diferenciar depressão e desmoralização $0^{40-44}$. De forma geral, a desmoralização estaria ligada ao sentimento de incompetência e de perda

Quadro 2. Estudos brasileiros que registraram a frequência de depressão pós-AVC

\begin{tabular}{|c|c|c|c|c|c|c|}
\hline Autor (ano) & Local & $\mathrm{n}$ & Contexto & Tempo pós-AVE & Instrumento & Frequência (\%) \\
\hline Simis e Nitrini $(2006)^{35}$ & Sorocaba (SP) & 93 & H & 2 semanas & HAM-D & $59,1^{\dagger}$ \\
\hline Carod-Artal et al. $(2009)^{34}$ & Brasília (DF) & $260^{*}$ & A & 20,7 meses (média) & HADS & $\begin{array}{c}20,0^{\dagger} \\
(\mathrm{F}: 25,0 ; \mathrm{M}: 15,4)\end{array}$ \\
\hline Terroni et al. $(2009)^{36}$ & São Paulo (SP) & 73 & $\mathrm{H} / \mathrm{A}$ & 1 semana -4 meses & HAM-D & $28,8^{\ddagger}$ \\
\hline Fróes et al. $(2011)^{32}$ & Fortaleza (CE) & 64 & $P R$ & $\begin{array}{c}<2 \text { anos: } 9,4 \% \\
2-6 \text { anos: } 50 \% \\
>7 \text { anos: } 40,7 \%\end{array}$ & $\mathrm{BDI}$ & $40,0^{+}$ \\
\hline Scheffer et al. $(2011)^{37}$ & Porto Alegre (RS) & $19^{*}$ & A & 9-27 meses & $\mathrm{BDI}$ & $33,3^{\dagger}$ \\
\hline Rangel et al. $(2013)^{38}$ & Maceió (AL) & $139^{*}$ & $P R$ & 3-316 meses & $\mathrm{BDI}$ & $49,7^{\dagger}$ \\
\hline
\end{tabular}

A: ambulatorial; BDI: Inventário de Depressão de Beck; F: gênero feminino; H: hospitalar; HADS: Escala Hospitalar de Ansiedade e Depressão; HAM-D: Escala de Depressão de Hamilton; PR: programa de reabilitação; M: gênero masculino. * 0 estudo incluiu pacientes com AVE hemorrágico (AVE isquêmico: Carod-Artal et al.: 87,7\%; Scheffer et al.: 84,2\%; Rangel et al.: 83,5\%). ${ }^{\dagger}$ Prevalência. ${ }^{\ddagger}$ Incidência em 4 meses. 
do autodomínio após fracassos repetidos, ao passo que a depressão se caracterizaria por anedonia e redução da motivação. Shader observou que o indivíduo com desmoralização pode responder favoravelmente a um estímulo positivo e ao alívio dos seus estressores, ao passo que o paciente com depressão não pode se livrar de seu estado negativo de humor, independentemente das mudanças ambientais ${ }^{45}$. O clínico, uma vez que reconheça a presença de desmoralização, deve trabalhar com o paciente para promover o sentimento de capacidade, domínio e retorno da esperança. Encorajamento, suporte e educação são essenciais.

Fedoroff et al. ${ }^{46}$, avaliando a adequação dos critérios diagnósticos para transtorno depressivo maior no diagnóstico de DPA, observaram que, exceto pela insônia terminal, todos os sintomas de depressão eram mais frequentes em pacientes com AVE e humor deprimido do que nos eutímicos. Contudo, alguns autores apontam para diferenças clínicas entre pacientes com DPA e aqueles com transtorno depressivo maior. Comparados a esses, os pacientes com AVE apresentam sinais mais graves de deterioração cognitiva, flutuações do humor, retardamento psicomotor, ansiedade e sintomas vegetativos e somáticos ${ }^{47}$. No Brasil, Terroni et al. ${ }^{36}$ chamam a atenção para a importância de sintomas de fadiga e de redução do interesse em geral no diagnóstico de DPA. Como não existem marcadores biológicos específicos, o diagnóstico é clínico e essa tarefa pode se tornar difícil na presença de déficits cognitivos graves, notadamente da linguagem.

A etiopatogênese da DPA é, provavelmente, multifatorial, o que explica a dificuldade de se encontrarem substratos neuroanatômicos precisos ${ }^{48-51}$. Pacientes que apresentam AVE vivenciam evento traumático que abala sua integridade corporal e mental, sua autonomia e sua estima pessoal, assim como suas vidas conjugal e profissional. Mecanismos psicológicos de adaptação, assim como personalidade pré-mórbida, certamente desempenham papel relevante no desenvolvimento da DPA. Além disso, as relações entre depressão e AVE podem ser ainda mais complexas: história de depressão aumenta cerca de 35\% o risco para o desenvolvimento de AVE, de maneira provavelmente independente de outros fatores de risco, como HAS e diabetes ${ }^{52}$.

Ainda que o debate se encontre aberto, as correlações entre local de lesão e sintomas depressivos são baixas. Os trabalhos de Robinson et al. sugerem que lesões anteriores, situadas próximas ao polo frontal esquerdo, estariam mais relacionadas ao desenvolvimento de sintomas depressivos ${ }^{8}$. Outros autores contestam isso ${ }^{11}$. Carson et al., em uma metanálise de 35 trabalhos que avaliaram a relação entre localização de lesões no sistema nervoso central e desenvolvimento de depressão, observaram que o risco para o desenvolvimento de depressão não estava associado com a localização das lesões ${ }^{49}$. Isso se deve possivelmente a vieses de seleção, dificuldade de examinar certos grupos de pacientes e varia- ções metodológicas. Ainda que a influência da lateralidade seja incerta, diversos trabalhos implicam disfunção frontoestriatal, ao menos nos primeiros meses após o AVE ${ }^{51}$.

O fator mais fortemente associado com o desenvolvimento de depressão é a gravidade da lesão inicial. Essa associação pode ser influenciada tanto por aspectos psicológicos, como reação afetiva desproporcional à gravidade do déficit, quanto biológicos, já que lesões maiores podem afetar mais circuitos cerebrais. Nesse sentido, Carota et al. ${ }^{53}$ observaram que a presença de labilidade emocional, especialmente choro, associada à gravidade inicial do déficit, estava entre os fatores preditivos mais relevantes para o desenvolvimento de depressão, até mais que a localização da lesão.

Além disso, pacientes que apresentam afasia não fluente grave, que são conscientes de seus déficits e que apresentam compreensão preservada vivenciam experiência extremamente angustiante. Eles mantêm, com esforço, fala espontânea reduzida, com poucas palavras, frases curtas e deficiências gramaticais. Dessa forma, como têm preservada a consciência de seus déficits, emerge enorme frustração por causa da dificuldade para falar. De fato, a afasia não fluente é considerada fator de risco para o desenvolvimento de depressão nas fases tardias do $A V E^{54}$. Em uma metanálise sobre riscos psicossociais para o desenvolvimento de DPA, Ouimet et al. observaram que, além de afasia, história pessoal de depressão ou de tratamentos psiquiátricos, incapacidade funcional, viver sozinho e isolamento social após o AVE se associam com aumento da probabilidade de DPA ${ }^{55}$.

Ainda que a patogênese da DPA seja discutida, é consenso que, se não tratada, exerce impacto negativo sobre a recuperação funcional ${ }^{56}$. No entanto, ainda não existem diretrizes para o tratamento e a efetividade das intervenções não está estabelecida. Hackett et al. concluíram que o uso de antidepressivos se associa a efeito benéfico pequeno, porém significativo ${ }^{57}$. Segundo Price et al., o uso de antidepressivos pode ser indicado tanto na abordagem da síndrome depressiva maior quanto na da menor ${ }^{58}$, porém não existem diretrizes para a escolha específica de fármacos. Os medicamentos mais estudados são os antidepressivos tricíclicos, em especial nortriptilina, e os inibidores seletivos da recaptação de serotonina (ISRS), especialmente fluoxetina, sertralina e citalopram. Existem, ainda, trabalhos avaliando a utilização de trazodona, venlafaxina, reboxetina, mirtazapina, milnaciprano e metilfenidato. De forma geral, Tharwani et al. recomendam o uso de nortriptilina, no entanto ressaltam seu perfil indesejável de efeitos colaterais e de interações farmacológicas ${ }^{59}$. Nesse caso, os ISRS surgem como alternativa. Mais polêmica e incerta é a utilização de antidepressivos na prevenção de DPA. Trabalhos isolados mostraram benefícios com nortriptilina, fluoxetina, sertralina, mirtazapina e metilfenidato, porém revisão sistemática da Cochrane não observou efeito significativo de antidepressivos na prevenção de DPA ${ }^{60}$. 
A abordagem psicoterápica associada ao uso de antidepressivos também parece se mostrar benéfica. Na metanálise de Hackett et al., um pequeno, mas significativo, efeito foi encontrado para a psicoterapia na prevenção da DPA60.

\section{Ansiedade}

Transtornos de ansiedade (TA) são frequentes após AVE, e entre $25 \%$ e $50 \%$ dos pacientes manifestam TAG nos primeiros meses, com pequena redução da incidência nos três anos seguintes ${ }^{61}$. Burton et al. observaram que TA diagnosticados por entrevista estruturada ou sintomas ansiosos mensurados por escalas específicas surgem em $20 \%$ a $25 \%$ dos pacientes em qualquer momento após AVE ${ }^{62}$. Segundo Angelelli et al..10, TA pós-AVE são observados em cerca de $23 \%$ dos indivíduos e aumentam consideravelmente entre pacientes com DPA, sugerindo que compartilhem mecanismos fisiopatológicos.

Os critérios diagnósticos do DSM-V para TA pós-AVE são aqueles do transtorno de ansiedade devido a uma condição médica geral, os quais correspondem aos critérios para pacientes sem lesão cerebral.

A maior parte dos estudos feitos com pacientes afetados por AVE no Brasil ou em outros países não explorou efetivamente a presença de TA. Assim, existem poucos dados sobre categorias específicas, como crises de pânico, agorafobia ou fobias. Segundo Burton et al., transtornos fóbicos e TAG são os tipos mais comuns de TA após um AVE22. Deve-se destacar que o TAG pós-AVE se aproxima em muitos aspectos do transtorno de estresse pós-traumático (TEPT), os dois manifestando-se após um evento imprevisível, capaz de ameaçar a vida do paciente. Recentemente, observou-se que o TEPT é relatado em cerca de $10 \%$ dos indivíduos afetados por AVE, enquanto a prevalência na população geral é de $1 \%$ a $2 \%{ }^{62}$. Além disso, são fatores de risco para desenvolvimento de TEPT: reação peritraumática intensa, gênero feminino e morbidade psiquiátrica prévia.

Assim como a DPA, os TA podem estar ligados a fatores psicológicos reacionais. As preocupações com a possibilidade de não controlar reações motoras, perceptivas, cognitivas e emocionais em diversos ambientes são frequentes no discurso dos pacientes com AVE, além do temor da ocorrência de novos eventos isquêmicos ${ }^{63}$. Isso pode se refletir na diminuição da percepção da QV28. Segundo Carod-Artal et al., pacientes com AVE mais grave e níveis elevados de ansiedade apresentavam pior QV ${ }^{34}$.

Por outro lado, os mecanismos neurobiológicos subjacentes aos fenômenos de ansiedade pós-AVE são pouco conhecidos. Os estudos que observaram a associação de ansiedade com sítio lesional não permitiram uma conclusão definitiva. As regiões cerebrais supostamente envolvidas fazem parte de circuitos frontossubcorticais (lobo frontal, tálamo, núcleos da base), do sistema límbico (amígdala, giro do cíngulo) e do eixo hipotálamo-hipofisário. Alguns estudos relataram associação entre síndromes depressivo-ansiosas e lesões corticais esquerdas, ao passo que pacientes somente com lesões subcorticais apresentavam depressão isolada. A ansiedade como sintoma isolado pareceria mais associada a lesões à direita ${ }^{64}$.

Uma revisão sistemática recente, que incluiu dois ensaios clínicos envolvendo 175 pacientes com diagnósticos de ansiedade e depressão pós-AVE comórbidos, encontrou evidências limitadas para o uso de psicofármacos no tratamento dos TA pós-AVE ${ }^{65}$. Os autores concluíram que o uso de paroxetina ou de buspirona pode ser eficaz no tratamento de sintomas ansiosos em pacientes com DPA. No entanto, ainda não há estratégias terapêuticas farmacológicas definitivas, havendo relato do uso de benzodiazepínicos por tempo limitado, de ISRS ou de buspirona ${ }^{31}$.

\section{Transtorno da expressão emocional involuntária e labilidade emocional}

Normalmente, rir e chorar são comportamentos desencadeados em contextos específicos e apropriados. No entanto, lesões do sistema nervoso central podem perturbar a regulação dessas manifestações emocionais. Após um AVE, elas podem aumentar em frequência, ser desencadeadas sem razões aparentes ou escapar ao controle habitual em contextos sociais, definindo transtornos específicos: o transtorno da expressão emocional involuntária (TEEI), também denominado riso e choro patológicos, afeto pseudobulbar ou riso e choro espasmódicos, que se distingue da labilidade emocional (ou emotionalism, em inglês) ${ }^{66}$.

TEEl associa-se habitualmente a sinais de comprometimento frontobulbar, como alterações da fala e da deglutição, e manifesta-se com caráter reflexo e espasmódico. Os episódios de choro ou de riso aparecem rapidamente após estímulos comuns ou até mesmo na ausência de contexto afetivo apropriado. Além disso, existe dissociação entre a expressão do afeto e o sentimento do paciente, de forma que ele ri ou chora sem sentir alegria ou tristeza, perdendo o controle de sua expressividade facial ${ }^{66,67}$.

Em contraste, os indivíduos com labilidade emocional não possuem o caráter forçado da expressão afetiva. Risos ou choros são provocados por estímulos que, de fato, têm um componente emocional e os sujeitos sentem a emoção apropriada (alegria ou tristeza), mas, tal como ocorre entre os pacientes com TEEl, não conseguem controlar sua expressão, exibindo um fenômeno de incontinência emocional ${ }^{66,67}$.

Todos esses comportamentos patológicos se distinguem dos risos e choros "normais" na medida em que são excessivos. No entanto, eles conservam seu componente social, uma vez que são desencadeados mais frequentemente em situações de caráter social do que quando o paciente está desacompanhado ${ }^{67}$. 
A maioria dos trabalhos sobre TEEl concentrou-se nos comportamentos de choro patológico. Segundo a literatura, ele ocorre em aproximadamente $7 \%$ dos casos nos primeiros dias após o AVE. Dois a quatro meses depois, a prevalência sobe para 18\% a 34\%, mantendo-se em 25\% após seis meses. Esses dados indicam que a prevalência tende a aumentar com o tempo, ao menos no que se refere aos seis primeiros meses. Como fatores de risco, foram descritos: AVC do tipo isquêmico, gênero feminino, sequelas motoras graves e disfunção cognitiva ${ }^{68-70}$.

Não se sabe se o TEEl e a labilidade emocional implicam disfunções neuronais diferentes. Além disso, essa distinção é frequentemente difícil de ser feita clinicamente, especialmente porque não há critérios definitivos para julgar a adequação emocional ou a frequência excessiva de risos e choros. No entanto, o substrato neuroanatômico parece ser mais claro para o TEEl do que para a labilidade emocional. 0 papel das estruturas pontinas ou mesencefálicas é sugerido no TEEl pela associação deste com lesões que possuem tal localização, de forma uni ou bilateral, e por relatos de tumores da fossa posterior ${ }^{66}$.

É importante considerar também fatores psicológicos que possam estar ligados aos transtornos da regulação afetiva $^{68}$. De fato, a labilidade emocional pode ser manifestação particular de TEPT e poderia ser, além disso, fator predisponente ao desenvolvimento de depressão, como relatado anteriormente. Tais transtornos podem gerar embaraço social e grande desconforto, levando o paciente a evitar interações sociais ou a apresentar ansiedade excessiva por causa da falta de autocontrole.

Em 2010, o Food and Drug Administration dos Estados Unidos aprovou o primeiro tratamento farmacológico para o TEEl, uma combinação de dextrometorfano e quinidina, comercializada com o nome comercial de Nuedexta ${ }^{\circledR 71}$. Em junho de 2013, essa combinação foi aprovada na União Europeia. No Brasil, essa medicação ainda não se encontra disponível. Como opções, estudos isolados relataram efeitos positivos de ISRS, antidepressivos tricíclicos ou agentes dopaminérgicos. Entre eles, citam-se amitriptilina, nortriptilina, desipramina, fluoxetina, citalopram, paroxetina, sertralina, levodopa e amantadina ${ }^{66,72,73}$.

\section{Irritabilidade, raiva e reação catastrófica}

Compreende-se raiva como uma emoção formada por componentes cognitivos, como indignação e hostilidade, e comportamentais, como agressividade, violência verbal ou física. Irritabilidade refere-se à condição de ser facilmente levado à raiva. Assim, a raiva apresenta-se como fenômeno complexo e multifatorial, envolvendo aspectos desenvolvimentais, socioculturais e fisiológicos ${ }^{74}$

As relações entre irritabilidade, raiva e AVE são bastante intrincadas. Adler et al. ${ }^{74}$ observaram que a ocorrência de AVE em homens era frequentemente precedida por estados afetivos negativos, especialmente raiva e desesperança. De forma similar, Gianturco et al. ${ }^{75}$ relataram que pacientes hospitalizados em decorrência de um AVE apresentavam mais frequentemente história de raiva precedendo a internação, em comparação com aqueles sem AVE.

Everson et al. ${ }^{76}$ relataram que homens propensos a exteriorizar raiva apresentavam risco duas vezes maior para AVE ou, caso tivessem doença coronariana, seis vezes maior, em comparação com aqueles menos propensos a manifestações de raiva. Williams et al. ${ }^{77}$ observaram que traços de personalidade raivosos aumentavam o risco de AVE, de forma mais significativa entre indivíduos mais jovens ou com hipercolesterolemia.

Angelelli et al. ${ }^{10}$ observaram que, no primeiro ano após um AVE, irritabilidade era uma das manifestações comportamentais mais frequentes. Ela se caracterizava mais por redução do limiar de tolerância do que por aumento das manifestações de raiva propriamente ditas. Paradiso et al. ${ }^{78}$ identificaram os seguintes fatores de risco: menor idade, sintomas depressivos, problemas cognitivos e lesões anteriores no hemisfério esquerdo. Além disso, essas dificuldades poderiam estar ligadas à presença de déficits motores, disartria e labilidade emocional.

Reações catastróficas representam um grau mais grave de comportamento associado à frustração ${ }^{31}$. Designam, no caso de pacientes afásicos, uma reação emocional intensa diante da incapacidade de executar uma tarefa linguística solicitada pelo examinador. $O$ paciente se sente impotente, manifestando-se por meio de choro, gestos e palavras de cólera $^{46}$. Ele geralmente se recusa a prosseguir o exame ou a conversação. Segundo Carota et al. ${ }^{79}$, cerca de 3,7\% dos pacientes apresentam reação catastrófica nos primeiros dias após um AVE. A maioria deles desenvolve labilidade emocional ou DPA nos meses seguintes, o que poderia sugerir uma conexão entre essas síndromes.

Assim como no TEEl, a vivência subjetiva e o comportamento podem estar dissociados. No caso da irritabilidade ou da agressividade, os pacientes frequentemente as banalizam e podem manifestá-las somente em situações específicas. No entanto, esses sintomas podem representar sobrecarga significativa e fonte de estresse para familiares e cuidadores $^{80}$. O impacto pode ser negativo na QV, pois a irritabilidade é um dos sintomas que mais levam os cuidadores a criticarem indivíduos que sofreram um AVE. Assim, é preciso estar atento ao desenvolvimento desses comportamentos.

Há poucos estudos abordando especificamente o tratamento desses sintomas. Carota et al. sugerem a utilização de abordagens cognitivo-comportamentais, por meio das quais os pacientes reconheçam sintomas iniciais de agressividade, assim como métodos de relaxamento ${ }^{79}$. Ressaltam, no entanto, que intervenções farmacológicas são frequentemente necessárias. Não há revisões sistemáticas ou metanálises sobre a utilização de psicofármacos nesse contexto. 
Chan et al. conduziram ensaio clínico para o tratamento de irritabilidade pós-AVE, utilizando fluoxetina e nortriptilina, e observaram que, apesar de a irritabilidade isoladamente não ter respondido, a redução de sintomas depressivos se associou à melhora significativa no comportamento agressivo ${ }^{80}$. De forma geral, as opções incluem antipsicóticos de primeira ou segunda geração, como haloperidol, olanzapina, quetiapina e risperidona; benzodiazepínicos; betabloqueadores; anticonvulsivantes, como carbamazepina e ácido valproico; além de fármacos serotoninérgicos, como ISRS, buspirona e trazodona ${ }^{80}$. Chama-se a atenção para potenciais problemas de interações farmacológicas e para a polêmica utilização de antipsicóticos em pacientes idosos, o que poderia associar-se ao aumento da mortalidade. Como exemplo da complexidade do manejo terapêutico desses casos, sabe-se que pacientes agitados recebem maior quantidade de medicação e tendem a sofrer mais quedas do que pacientes não agitados.

Com relação à abordagem das reações catastróficas, as melhores estratégias são preventivas, resguardando o paciente de situações que o predisponham aos episódios ${ }^{64}$, durante os quais o manejo se baseia na abordagem acolhedora e segura, transmitindo tranquilidade até que a exaltação ceda.

\section{Apatia}

A apatia é comumente definida como uma síndrome de diminuição de comportamentos, emoções e cognições direcionados a um objetivo ${ }^{81}$. A utilização de questionários padronizados demonstrou prevalências de apatia que variam de $20 \%$ a $50 \%$ nos primeiros meses após um AVE ${ }^{26,82}$. Nas fases mais agudas, a apatia é frequente entre os pacientes com AVE à direita, em especial no território da artéria cerebral média ${ }^{63}$. Angelelli et al. ${ }^{10}$, no entanto, relataram que a apatia é um fenômeno que tende a se manifestar especialmente na fase crônica pós-AVE, acometendo cerca de $27 \%$ dos casos. Recentemente, Van Dalen et al. observaram prevalência média de 34,6\%, equiparando-se à ocorrência de depressão ou de síndromes demenciais pós-AVE. Os autores salientam que, enquanto depressão e demência pós-AVE são frequentemente estudadas, a apatia pós-AVE tem sido negligenciada ${ }^{81}$.

Do ponto de vista comportamental, são classificados como apáticos os pacientes que apresentam redução da iniciativa verbal ou motora, com diminuição do comportamento voluntário e intencional ${ }^{81}$. De forma mais sutil, eles também manifestam achatamento afetivo, que se associa à perda de motivação e de vontade. A apatia, sem dúvida, pode fazer parte de uma síndrome depressiva, no entanto, no caso de associação com depressão, existe sofrimento subjetivo evidente, com manifestação de outros sintomas, como tristeza, baixa autoestima, culpabilidade, ideação negativa e de morte, e labilidade emocional. Além disso, há na depressão a tendência de interpretar negativamente quais- quer estímulos de conteúdo emocional, sejam eles neutros ou positivos. Com efeito, a apatia secundária ao AVE não se acompanha necessariamente de sofrimento subjetivo ou de ideação negativa sobre si mesmo. Em sua metanálise, Van Dalen et al. observaram que depressão estava presente em 40,1\% dos pacientes com apatia, confirmando a associação, mas também a independência, das duas síndromes ${ }^{81}$.

Em geral, a apatia se associa à idade mais avançada, sendo concomitante a sinais, como perseveração, síndrome de dependência ambiental e liberação de reflexos primitivos ${ }^{81-83}$. Van Dalen et al. observaram que a apatia pós-AVE estava associada a pior funcionamento cognitivo ${ }^{81}$. De forma sintética, a apatia associada a lesões cerebrais se manifesta por reatividade afetiva reduzida a qualquer estímulo de conteúdo emocional, seja sua natureza positiva, negativa ou neutra ${ }^{81}$.

Todos os estudos clínicos sugerem que a apatia é ligada à disfunção de circuitos frontossubcorticais ${ }^{83}$. Esses incluem a região dorsolateral do lobo frontal, o giro do cíngulo, a área motora suplementar, os núcleos da base e o núcleo dorsomediano do tálamo, entre outros. Os estudos de neuroimagem funcional realizados em pacientes com apatia grave pós-AVE mostraram redução do metabolismo pré-frontal dorsolateral e temporal ${ }^{82}$. Van Dalen et al., no entanto, não observaram associações entre localização ou volume das lesões, nem gravidade inicial do AVE com o desenvolvimento de apatia ${ }^{81}$.

Os mecanismos subjacentes à apatia podem ser divididos em três subtipos, com base em distúrbios do processamento emocional, cognitivo ou de autoativação ${ }^{83}$, como mostra o quadro 3. O último é responsável pela forma mais grave, chamada síndrome de déficit de autoativação, ou atimormia, caracterizada por "esvaziamento mental". Os pacientes podem apresentar atividade adequada após estimulação repetida, demonstrando a dissociação entre autoativação perturbada e heteroativação intacta. Essa síndrome sugere que lesões diretas da eferência dos núcleos da base (globo pálido interno ou vias estriato-palidais) resultam em perda da amplificação de sinais relevantes, diminuindo-os no córtex frontal.

Diversos pesquisadores propuseram que apatia, abulia e mutismo acinético seriam estados clínicos caracterizados por comportamento reduzido, formando um continuum no qual os extremos incluiriam a abulia e o mutismo acinético. Neste, os pacientes podem ser incapazes de iniciar movimentos ou de falar ${ }^{84}$. Eles podem estar incontinentes e comer ou beber somente se estimulados energicamente por um terceiro. Quando falam, é somente por monossílabos e não manifestam nenhuma emoção, mesmo em caso de dor.

De acordo com Van Dalen et al., a heterogeneidade dos trabalhos incluídos em sua revisão sistemática dificultou a avaliação quantitativa da relação entre apatia e recuperação funcional ${ }^{81}$. No entanto, observou-se a tendência de que a apatia possa ter impacto negativo na reabilitação dos pacientes, mesmo moderada. 
Quadro 3. Características dos mecanismos subjacentes à apatia

\begin{tabular}{|c|c|c|}
\hline Subtipos de apatia & Características clínicas & Localização \\
\hline Distúrbios do processamento emocional & $\begin{array}{l}\text { Incapacidade de estabelecer as conexões necessárias entre sinais } \\
\text { afetivos e o comportamento presente ou futuro }\end{array}$ & $\begin{array}{l}\text { Lesões do córtex pré-frontal orbitomedial ou de regiões límbicas } \\
\text { nos núcleos da base (por exemplo, estriado ventral ou globo } \\
\text { pálido ventral) }\end{array}$ \\
\hline Distúrbios do processamento cognitivo & $\begin{array}{l}\text { Dificuldades na elaboração do plano de ações para } 0 \\
\text { comportamento presente ou futuro }\end{array}$ & $\begin{array}{l}\text { Lesões do córtex pré-frontal dorsolateral ou de áreas associativas } \\
\text { nos núcleos da base (por exemplo, núcleo caudado dorsal) }\end{array}$ \\
\hline Distúrbios da autoativação & $\begin{array}{l}\text { Incapacidade de autoativar pensamentos ou autoiniciar ações, } \\
\text { contrastando com habilidade relativamente preservada para } \\
\text { iniciar comportamentos externamente dirigidos }\end{array}$ & $\begin{array}{l}\text { Lesões bilaterais, afetando territórios associativos e límbicos da } \\
\text { porção interna do globo pálido }\end{array}$ \\
\hline
\end{tabular}

Até o momento, não foram conduzidos ensaios clínicos sistemáticos e não existem evidências suficientes para indicar qualquer tratamento farmacológico específico. Há relatos de benefícios com alguns fármacos, que talvez mereçam maior investigação. Dentre eles, destacam-se nefiracetam, donepezil, agonistas dopaminérgicos, como a bromocriptina, e metilfenidato. Os ISRS parecem não exercer efeitos significativos. Programas de reabilitação envolvendo terapeutas ocupacionais, neuropsicológos e treinamento de cuidadores, por exemplo, podem ser adaptados de acordo com as necessidades dos pacientes ${ }^{81}$.

\section{Demência}

A demência vascular (DV) é a segunda causa de demência na população geral, podendo ter sua prevalência subestimada em virtude da frequente associação com doença de Alzheimer $(D A)^{85}$. A prevalência estimada de DV tende a aumentar com a idade, variando entre 1,2\% e 4,2\% aos 65 anos e entre $3,5 \%$ e 5,8\% aos 90 anos $^{86}$. A prevalência de DV em um centro terciário de atenção a pacientes com demências, em Ribeirão Preto, foi de $25,8 \%^{87}$. Inúmeros estudos investigaram a incidência de comprometimento cognitivo e de demência pós-AVE, encontrando taxas de $6 \%$ a 32\% em pacientes acompanhados por 20 anos $^{88,89}$

O termo DV engloba diversos processos patológicos, que incluem doenças de pequenos ou de grandes vasos, infartos recorrentes ou, de forma controversa, estratégicos, e hipoperfusão subcortical crônica ${ }^{86}$. Entre os fatores de risco, encontram-se idade avançada, gravidade inicial do evento isquêmico, HAS, obesidade, diabetes, fibrilação atrial, evidências radiológicas de lesões de substância branca ou de atrofia cortical (especialmente no lobo temporal) e hiper-homocisteinemia. Além disso, trabalhos demonstram que déficits cognitivos pré-mórbidos também são fatores de risco, ao passo que altos índices de escolaridade podem diminuir o risco de declínio cognitivo pós-AVE ${ }^{86}$.

Em função da heterogeneidade neuropatológica, há também grande diversidade das manifestações clínicas, dependendo da natureza, da localização e da extensão das lesões. Tradicionalmente, reconhecem-se dois padrões clínicos principais: um no qual o comprometimento predominante é cortical e o outro, subcortical. Embora esses padrões ainda sejam internamente heterogêneos, ambos apresentam características clínicas distintas entre $\mathrm{si}^{85}$.

$\mathrm{Na}$ DV cortical, as alterações cognitivas tendem a refletir as áreas corticais afetadas. Em geral, decorrem da oclusão de ramos arteriais corticais, frequentemente causados por fenômenos aterotrombóticos ou tromboembólicos a partir do coração ou de grandes vasos, podendo se expressar clinicamente como AVE. Assim, lesões frontomediais podem cursar com disfunção executiva, abulia ou apatia, além de mutismo acinético no caso de comprometimento bilateral. Lesões do lobo parietal esquerdo podem levar a afasia, apraxia ou agnosia, ao passo que lesões do lobo parietal direito podem cursar com síndrome de heminegligência, confusão mental, agitação psicomotora, déficits visuoespaciais e de habilidades visuoconstrutivas. Lesões da região temporal medial podem levar à amnésia anterógrada. O surgimento da síndrome demencial pode dar-se, então, de forma súbita. Quando a divisão superior da artéria cerebral média não é envolvida, sinais motores focais podem não aparecer e a instalação súbita de alterações cognitivas isoladas talvez não seja óbvia clinicamente. Assim, seu curso pode ser insidioso e não é incomum que o paciente se recupere até o próximo evento isquêmico. O curso é percebido como flutuante ou em degraus. Cerca de 30\% dos pacientes com demência multi-infarto experimentam tanto início abrupto quanto progressão em degraus.

Já na DV subcortical, os mecanismos principais se associam a alterações dos pequenos vasos. Dentre eles, destacam-se infartos lacunares e isquemia subcortical crônica, afetando principalmente os núcleos da base e a substância branca, de forma silenciosa. Frequentemente interrompem circuitos frontais e outros circuitos córtico-corticais, levando a manifestações clínicas atribuíveis a áreas cerebrais remotas (distantes) às lesões. As principais características clínicas incluem sinais motores focais, distúrbios da marcha (marcha com pequenos passos ou parkinsoniana, apraxia de marcha), história de desequilíbrio e de quedas frequentes e não provocadas, aumento da frequência urinária, com urgência e outros sintomas urinários não urológicos, alterações de personalidade e de humor, apatia, incontinência emocional, transtorno cognitivo caracterizado por déficit de memória relativamente leve, lentificação psicomotora e disfunção exe- 
cutiva. O curso da DV subcortical pode ser gradual ou em degraus, podendo ser também de progressão lenta ou rápida.

A presença de doença cerebrovascular e de fatores de risco vasculares associa-se a aumento de risco e de aceleração da $\mathrm{DA}^{90}$. No entanto, sintomas depressivos e labilidade emocional são mais frequentes na DV do que na DA ${ }^{91}$. A descoberta da associação entre o aparecimento de uma síndrome depressiva na população geriátrica e a presença de lesões da substância branca de origem vascular, sobretudo nas regiões frontais, levou à hipótese da existência de uma "depressão vascular", que estaria ligada à microangiopatia.

Não existem tratamentos específicos para o déficit cognitivo da DV ${ }^{92}$. Pacientes com comprometimento cognitivo e evidências de doença cerebrovascular devem ter fatores de risco investigados e tratados, especialmente HAS. As evidências de déficit colinérgico na DV similares àqueles da DA têm levado ao estudo dos inibidores da colinesterase. A resposta a esses fármacos é variável, com alta proporção de indivíduos sem resposta ${ }^{92}$. Naqueles em que houve resposta, seus benefícios cognitivos e funcionais tiveram magnitude similar aos daqueles descritos para a DA, representando um atraso de quatro a seis meses no declínio cognitivo, e isso pode se dever à associação neuropatológica com a DA. A memantina mostrou efeitos benéficos em escalas cognitivas, que não foram traduzidos na impressão clínica global ou no desempenho de AVD em pacientes com DV.

\section{Mania}

A ocorrência de episódios maníacos associados a AVE é rara, não chegando a 1\% dos casos. Santos et al. relataram que, nos últimos 50 anos, apenas 74 de casos de mania pós-AVE foram publicados ${ }^{93}$. Essa baixa prevalência limita a descrição das características clínicas, demográficas e prognósticas dessa síndrome. No entanto, com os dados disponíveis, é possível observar algumas características consistentes.

Predomina no gênero masculino, sem influência da idade, de história pessoal ou familiar de transtorno psiquiátrico ou de atrofia subcortical ${ }^{93}$. Adicionalmente, acompanha-se de sinais neurológicos ou cognitivos ligados à localização da lesão (por exemplo, heminegligência ou síndrome disexecutiva). A maior parte dos relatos associa-se a lesões no hemisfério direito, incluindo lobos frontais ou temporais, tálamo ou núcleo caudado. Nesse sentido, Starkstein et al. ${ }^{94}$ observaram hipometabolismo da região temporal inferior direita em três pacientes com mania associada a lesões subcorticais. Os autores sugerem, como mecanismo fisiopatológico, disfunção da alça fronto-estriado-tálamo-cortical interconectada ao sistema límbico e ao lobo temporal, principalmente o direito. É interessante notar a oposição entre episódios depressivos secundários a lesões esquerdas e episódios maníacos a lesões direitas.

A maior parte dos casos tende a surgir no primeiro mês, havendo, no entanto, casos iniciados até dois anos pós-AVE ${ }^{93}$.
Aproximadamente, 92\% dos pacientes apresentam elevação do humor como primeiro sintoma ${ }^{93}$. Cerca de $41 \%$ apresentam oscilação entre humor elevado e disforia, e apenas 8\%, disforia isolada. Os episódios duram de 1 a 12 semanas, frequentemente sem recorrências ou hipomania residual.

A baixa prevalência dificulta o tratamento baseado em evidências ${ }^{93}$. A literatura mostra que o lítio é eficaz. Outros estabilizadores do humor, como ácido valproico e carbamazepina, também têm sido utilizados. Antipsicóticos vêm sendo usados em casos de mania grave com sintomas psicóticos. Benzodiazepínicos também têm sido usados como adjuvantes no tratamento da hiperatividade e da insônia.

\section{Síndromes psicóticas}

Síndromes psicóticas também são complicações raras após AVE. Rabins et al..$^{95}$ avaliaram, durante nove anos, todos os indivíduos com mais de 60 anos admitidos em um hospital, identificando somente cinco pacientes com psicose pós-AVE em 301 casos (0,02\%). Todos eles apresentavam lesões frontoparietais à direita e mostravam grau significativo de atrofia subcortical em comparação com pacientes pareados com AVE, porém sem psicose.

É interessante notar que a maior parte dos relatos de casos de transtornos psicóticos pós-AVE é caracterizada por delírios monotemáticos de identificação, como síndrome de Capgras, síndrome de Fregoli, paramnésia reduplicativa ou intermetamorfose, e associa-se a lesões no hemisfério direito ${ }^{96-98}$. Na paramnésia reduplicativa, já se observaram lesões localizadas nas regiões frontais, parietais, talâmicas ou no lobo temporal médio99. Já os casos de síndrome de Capgras pós-AVE mostram predominância de lesões no lobo frontal direito ou na junção parieto-occipital direita ${ }^{100-102}$. Além disso, no trabalho de Rabins et al.95, o desenvolvimento de sintomas psicóticos pareceu associar-se à ocorrência de crises epilépticas. Em geral, os pacientes com sintomas psicóticos respondem ao tratamento com fármacos antipsicóticos ${ }^{95}$.

\section{CONCLUSÃO}

Tendo em vista a magnitude do problema de saúde pública relacionado ao AVE e sua frequente associação a transtornos neuropsiquiátricos, é notória a escassez de dados sobre seu manejo terapêutico. Uma multiplicidade de alterações comportamentais e afetivas pode associar-se a lesões vasculares do sistema nervoso central e se sobrepõem com frequência a déficits cognitivos, interferindo nos programas de reabilitação e de reinserção social. Além da intervenção precoce para prevenir o infarto cerebral, o aprofundamento do conhecimento sobre os transtornos neuropsiquiátricos parece ter grande potencial para melhorar o prognóstico e a QV de pacientes que sofreram um AVE. 


\section{CONTRIBUIÇÕES INDIVIDUAIS}

Todos os autores contribuíram efetivamente na concepção do presente trabalho, no levantamento de dados e em sua análise e interpretação. Participaram significativamente na redação do artigo, revisaram criticamente o seu conteúdo e aprovaram a versão final a ser publicada.

\section{AGRADECIMENTO}

Este trabalho foi financiado parcialmente por Fundação de Amparo à Pesquisa do Estado de Minas Gerais (Fapemig) e Conselho Nacional de Desenvolvimento Científico e Tecnológico (CNPq).

\section{CONFLITOS DE INTERESSES}

Os autores declaram não possuir conflitos de interesses.

\section{REFERÊNCIAS}

1. World Health Organization. Recommendations on stroke prevention, diagnosis, and therapy: report of the WHO Task Force on Stroke and Other Cerebrovascular Disorders. Stroke. 1989;20(10):1407-31.

2. Broderick JP, Philips SJ, Whisnat JP, O'Fallon WM, Bergstralh EJ. Incidence rates of strokes in the eighties: the decline in stroke. Stroke. 1989;20(5):577-82.

3. Heuschmann PU, Kolominsky-Rabas PL, Misselwitz B, Hermanek P, Leffmann C, Janzen RW, et al. Predictors of in-hospital mortality and attributable risks of death after ischemic stroke: the German stroke registers study group. Arch Intern Med. 2004;164(16):1761-8.

4. de Carvalho JJ, Alves MB, Viana GA, Machado CB, dos Santos BF, Kanamura AH, et al. Stroke epidemiology, patterns of management, and outcomes in Fortaleza, Brazil: a hospitalbased multicenter prospective study. Stroke. 2011;42(12):3341-6.

5. Minelli C, Fen LF, Minelli DP. Stroke incidence, prognosis, 30-day, and 1-year case fatality rates in Matão, Brazil: a population-based prospective study. Stroke. 2007;38(11):2906-11.

6. Rabelo DF, Néri AL. Bem-estar subjetivo e senso de ajustamento psicológico em idosos que sofreram acidente vascular cerebral: uma revisão. Estud Psicol (Natal). 2006;11(2):169-77.

7. Mota JF, Nicolato R. Qualidade de vida em sobreviventes de acidente vascular cerebral: instrumentos de avaliação e seus resultados. J Bras Psiquiatr. 2008;57(2):148-56

8. Chemerinski E, Robinson RG. The neuropsychiatry of stroke. Psychosomatics. 2000;41(1):5-14.

9. Tengs TO, Yu M, Luistro E. Health-related quality of life after stroke - a comprehensive review. Stroke. 2001;32(4):964-72.

10. Angelelli P, Paolucci S, Bivona U, Piccardi L, Ciurli P, Cantagallo A, et al. Development of neuropsychiatric symptoms in poststroke patients: a cross-sectional study. Acta Psychiatr Scand. 2004;110(1):55-63.

11. Robinson RG, Starr LB, Kubos KL, Price TR. A two-year longitudinal study of post-stroke mood disorders: findings during the initial evaluation. Stroke. 1983;14(5):736-41.

12. Robinson RG, Starr LB, Price TR. A two year longitudinal study of mood disorders following stroke. Prevalence and duration at six months follow-up. Br J Psychiatry. 1984;144:256-62.

13. Robinson RG, Bolduc PL, Price TR. Two-year longitudinal study of poststroke mood disorders: diagnosis and outcome at one and two years. Stroke. 1987;18(5):837-43.

14. Eastwood MR, Rifat SL, Nobbs H, Ruderman J. Mood disorder following cerebrovascular accident. Br J Psychiatry. 1989;154:195-200.

15. Morris PLP, Robinson RG, Raphael B. Prevalence and outcome of poststroke depression in hospitalized patients. Int J Psychiatr Med. 1990;20(4):327-42.

16. House A, Dennis M, Mogridge L, Warlow C, Hawton K, Jones L. Mood disorders in the year after stoke. Br J Psychiatry. 1991;158:83-92
17. Astrom M, Adolfsson R, Asplund K. Major depression in stroke patients: a 3-year longitudinal study. Stroke. 1993;24(7):976-82.

18. Burvill PW, Johnson GA, Jamrozik KD, Anderson CS, Stewart-Wynne EG, Chakera TM. Prevalence of depression after stroke: the Perth Community Stroke Study. Br J Psychiatry. 1995:166(3):320-7.

19. $\mathrm{Ng} \mathrm{KC}$, Chan KL, Straughan PT. A study of post-stroke depression in a rehabilitative center. Acta Psych Scand. 1995;92(1):75-9.

20. Kotila M, Numminen $H$, Waltimo 0 , Kaste $M$. Depression after stroke: results of the FINNSTROKE Study. Stroke. 1998;29(2):368-72.

21. Hackett ML, Anderson CS. Predictors of depression after stroke: a systematic review of observational studies. Stroke. 2005;36(10): 2296-301.

22. Buijck BI, Zuidema SU, Spruit-van Eijk M, Geurts AC, Koopmans RT. Neuropsychiatric symptoms in geriatric patients admitted to skilled nursing facilities in nursing homes for rehabilitation after stroke: a longitudinal multicenter study. Int J Geriatr Psychiatry. 2012;27(7):734-41.

23. Ayerbe $L$, Ayis S, Wolfe CD, Rudd AG. Natural history, predictors and outcomes of depression after stroke: systematic review and meta-analysis. Br J Psychiatry. 2013;202(1):14-21.

24. Ayerbe L, Ayis S, Crichton S, Wolfe CD, Rudd AG. The natural history of depression up to 15 years after stroke: the South London Stroke Register. Stroke. 2013;44(4):1105-10.

25. Skånér Y, Nilsson GH, Sundquist K, Hassler E, Krakau I. Self-rated health, symptoms of depression and general symptoms at 3 and 12 months after a first-ever stroke: a municipality-based study in Sweden. BMC Fam Pract. 2007;8:61.

26. Starkstein SE, Fedoroff JP, Price TR, Leiguarda R, Robinson RG. Apathy following cerebrovascular lesions. Stroke. 1993;24(11):1625-30.

27. Brodaty H, Sachdev PS, Withall A, Altendorf A, Valenzuela MJ, Lorentz L. Frequency and clinical, neuropsychological and neuroimaging correlates of apathy following stroke - the Sydney Stroke Study. Psychol Med. 2005;35(12):1707-16.

28. Campbell Burton CA, Murray J, Holmes J, Astin F, Greenwood D, Knapp P. Frequency of anxiety after stroke: a systematic review and meta-analysis of observational studies. Int J Stroke. 2013:8(7):545-59.

29. Calvert T, Knapp P, House A. Psychological associations with emotionalism after stroke. J Neurol Neurosurg Psychiatry. 1998;65(6):928-9.

30. Piamarta F, lurlaro S, Isella V, Atzeni L, Grimaldi M, Russo A, et al. Unconventional affective symptoms and executive functions after stroke in the elderly. Arch Gerontol Geriatr Suppl. 2004;(9):315-23.

31. Robinson RG. The clinical neuropsychiatry of stroke. New York: Cambridge University Press; 2006.

32. Fróes KSSO, Valdés MTM, Lopes DPLO, Silva CEP. Factors associated with health-related quality of life for adults with stroke sequelae. Arq Neuropsiquiatr. 2011;69(2B):371-6.

33. Souza $A C$, Rocha MO, Teixeira AL, Dias Júnior J0, Sousa LA, Nunes MC. Depressive symptoms and disability in chagasic stroke patients: impact on functionality and quality of life. J Neurol Sci. 2013;324(1-2):34-7.

34. Carod-Artal FJ, Trizotto DS, Coral LF, Moreira CM. Determinants of quality of life in Brazilian stroke survivors. J Neurol Sci. 2009;284(1-2):63-8.

35. Simis S, Nitrini R. Cognitive improvement after treatment of depressive symptoms in the acute phase of stroke. Arg Neuropsiquiatr. 2006;64(2B):412-7.

36. Terroni LM, Fráguas R, Lucia M, Tinone G, Mattos P, losifescu DV, et al. Importance of retardation and fatigue/interest domains for the diagnosis of major depressive episode after stroke : a four months prospective study. Rev Bras Psiquiatr. 2009;31(3):202-7.

37. Scheffer M, Monteiro JK, de Almeida RMM. Frontal stroke: problem solving, decision making, impulsiveness, and depressive symptoms in men and women. Psychol Neurosci. 2011;4(2):267-78

38. Rangel ESS, Belasco AGS, Diccini S. Qualidade de vida de pacientes com acidente vascular cerebral em reabilitação. Acta Paul Enferm. 2013;26(2):205-12.

39. Lyketsos CG, Kozauer N, Rabins PV. Psychiatric manifestations of neurologic disease: where are we headed? Dialogues Clin Neurosci. 2007;9(2):111-24.

40. Schildkraut JJ, Klein FD. The classification and treatment of depressive states. In: Shader RI. Manual of psychiatric therapeutics. Boston: Little and Brown; 1975.

41. Figueiredo JM, Frank JD. Subjective incompetence, the clinical hallmark of demoralization. Compr Psychiatry. 1982;23(4):353-63.

42. Figueiredo JM. Some issues in research on the epidemiology of demoralization. Compr Psychiatry. 1983;24(2):154-7.

43. Figueiredo JM. Depression and demoralization: phenomenologic differences and research perspectives. Compr Psychiatry. 1993;34(5):308-11.

44. Clarke DM, Kissane DW. Demoralization: its phenomenology and importance. Aust NZJ Psychiatry. 2002;36(6):733-42 
45. Shader RI. Demoralization revisited. J Clin Psychopharmacology. 2005;25(4):291-2.

46. Fedoroff JP, Starkstein SE, Parikh RM, Price TR, Robinson RG. Are depressive symptoms nonspecific in patients with acute stroke? Am J Psychiatry. 1991;148(9):1172-6.

47. Gainotti G, Azzoni A, Marra C. Frequency, phenomenology and anatomical-clinical correlates of major post-stroke depression. Br J Psychiatry. 1999;175:163-7.

48. Bhogal SK, Teasell R, Foley N, Speechley M. Lesion location and poststroke depression: systematic review of the methodological limitations in the literature. Stroke. 2004;35(3):794802.

49. Carson AJ, MacHale S, Allen K, Lawrie SM, Dennis M, House A, et al. Depression after stroke and lesion location: a systematic review. Lancet. 2000;356(9224):122-6.

50. MacHale SM, O'Rourke SJ, Wardlaw JM, Dennis MS. Depression and its relation to lesion location after stroke. J Neurol Neurosurg Psychiatry. 1998;64(3):371-4.

51. Shimoda K, Robinson RG. The relationship between poststroke depression and lesion location in long-term follow-up. Biol Psychiatry. 1999;45(2):187-92.

52. Dong JY, Zhang YH, Tong J, Qin LQ. Depression and risk of stroke: a meta-analysis of prospective studies. Stroke. 2012;43(1):32-7.

53. Carota A, Berney A, Aybek S, laria G, Staub F, Ghika-Schmid F, et al. A prospective study of predictors of poststroke depression. Neurology. 2005;64(3):428-33.

54. Kauhanen ML, Korpelainen JT, Hiltunen P, Määttä R, Mononen H, Brusin E, et al. Aphasia, depression, and non-verbal cognitive impairment in ischaemic stroke. Cerebrovasc Dis. 2000;10(6):455-61.

55. Ouimet MA, Primeau F, Cole MG. Psychosocial risk factors in poststroke depression: a systematic review. Can J Psychiatry. 2001;46(9):819-28.

56. Ribeiro DS, Cerqueira Filho EA, Losapio MF, Sena EP. Terapia medicamentosa na depressão pós-acidente vascular encefálico. J Bras Psiquiatr. 2009;58(2):135-42.

57. Hackett ML, Anderson CS, House A0, Xia J. Interventions for treating depression after stroke. Stroke. 2009;40:e487-8.

58. Price A, Rayner L, Okon-Rocha E, Evans A, Valsraj K, Higginson IJ, et al. Antidepressants for the treatment of depression in neurological disorders: a systematic review and metaanalysis of randomised controlled trials. J Neurol Neurosurg Psychiatry. 2011;82(8):914-23.

59. Tharwani HM, Yerramsetty P, Mannelli P, Patkar A, Masand P. Recent advances in poststroke depression. Curr Psychiatry Rep. 2007;9(3):225-31.

60. Hackett ML, Anderson CS, House A, Halteh C. Interventions for preventing depression after stroke. Cochrane Database Syst Rev. 2008;(3):CD003689.

61. Astrom M. Generalized anxiety disorder in stroke patients. A 3-year longitudinal study. Stroke. 1996:27(2):270-5

62. Favrole $P$, Jehel $L$, Levy $P$, Descombes $S$, Muresan IP, Manifacier MJ, et al. Frequency and predictors of post-traumatic stress disorder after stroke: a pilot study. J Neurol Sci. 2013;327(1-2):35-40.

63. Carota A, Dieguez S, Bogousslavsky J. Psychopathology of stroke. Psychol Neuropsychiatr Vieil. 2005;3(4):235-49.

64. Robinson RG. Neuropsychiatric consequences of stroke. Annu Rev Med. 1997;48:217-29.

65. Burton CAC, Holmes J, Murray J, Gillespie D, Lightbody CE, Watkins CL, et al. Interventions for treating anxiety after stroke. Cochrane Database Syst Rev. 2011;(12):CD008860.

66. Sartori HCS, Barros T, Tavares A. Transtorno da expressão emocional involuntária. Rev. Psiq Clín. 2008;35(1):20-5.

67. Grinblat N, Grinblat E, Grinblat J. Uncontrolled crying: characteristics and differences from normative crying. Gerontology. 2004;50(5):322-9.

68. Kim JS, Choi-Kwon S. Poststroke depression and emotional incontinence: correlation with lesion location. Neurology. 2000;54(9):1805-10.

69. Tang WK, Chan SS, Chiu HF, Ungvari GS, Wong KS, Kwok TC. Emotional incontinence in Chinese stroke patients: diagnosis, frequency, and clinical and radiological correlates. J Neurol. 2004;251(7):865-9.

70. House A, Dennis M, Molyneux A, Warlow C, Hawton K. Emotionalism after stroke. BMJ. 1989;298(6679):991-4.

71. Pioro EP, Brooks BR, Cummings J, Schiffer R, Thisted RA, Wynn D, et al. Dextromethorphan plus ultra low-dose quinidine reduces pseudobulbar affect. Ann Neurol. 2010;68(5):693-702.

72. Robinson RG, Parikh RM, Lipsey JR, Starkstein SE, Price TR. Pathological laughing and crying following stroke: validation of measurement scale and double-blind treatment study. Am J Psychiatry. 1993;150(2):286-93.

73. Andersen G, Vestergaard K, Riis J. Citalopram for post-stroke pathological crying. Lancet. 1993;342(8875):837-9.

74. Adler R, MacRitchie K, Engel GL. Psychologic processes and ischemic stroke (occlusive cerebrovascular disease), I: observations on 32 men with 35 strokes. Psychosom Med. $1971 ; 33(1): 1-29$
75. Gianturco DT, Breslin MS, Heyman A, Gentry WD, Jenkins CD, Kaplan B. Personality patterns and life stress in ischemic cerebrovascular disease, I: psychiatric findings. Stroke. 1974;5(4):453-60.

76. Everson SA, Kaplan GA, Goldberg DE, Lakka TA, Sivenius J, Salonen JT. Anger expression and incident stroke: prospective evidence from the Kuopio Ischemic Heart Disease Study. Stroke. 1999;30(3):523-8.

77. Williams JE, Nieto FJ, Sanford (P, Couper DJ, Tyroler HA. The association between trait anger and incident stroke risk: the Atherosclerosis Risk in Communities (ARIC) Study. Stroke. 2002;33(1):13-9.

78. Paradiso S, Robinson RG, Arndt S. Self-reported aggressive behavior in patients with stroke. J Nerv Ment Dis. 1996;184(12):746-53.

79. Carota A, Rossetti A0, Karapanayiotides T, Bogousslavsky J. Catastrophic reaction in acute stroke : a reflex behavior in aphasic patients. Neurology. 2001;57(10):1902-5.

80. Chan KL, Campayo A, Moser DJ, Arndt S, Robinson RG. Aggressive behavior in patients with stroke: association with psychopathology and results of antidepressant treatment on aggression. Arch Phys Med Rehabil. 2006;87(6):793-8.

81. van Dalen JW, Moll van Charante EP, Nederkoorn PJ, van Gool WA, Richard E. Poststroke apathy. Stroke. 2013;44(3):851-60.

82. Okada K, Kobayashi S, Yamagata S, Takahashi K, Yamaguchi S. Poststroke apathy and regional cerebral blood flow. Stroke. 1997;28(12):2437-41.

83. Levy R, Dubois B. Apathy and the functional anatomy of the prefrontal cortex-basal ganglia circuits. Cereb Cortex. 2006;16(7):916-28.

84. Nagaratnam N, Nagaratnam K, Ng K, Diu P. Akinetic mutism following stroke. J Clin Neurosci. 2004;11(1):25-30.

85. Wallin A, Milos V, Sjogren M, Pantoni L, Erkinjuntti T. Classification and subtypes of vascular dementia. Int Psychogeriatr. 2003;15(Suppl 1):27-37.

86. Hébert R, Brayne C. Epidemiology of vascular dementia. Neuroepidemiology. 1995;14(5):240-57.

87. Vale FC, Miranda SJC. Clinical and demographic features of patients with dementia attended in a tertiary outpatient clinic. Arq Neuropsiquiatr. 2002;60(3-A):548-52.

88. Béjot Y, Aboa-Eboulé C, Durier J, Rouaud 0, Jacquin A, Ponavoy E, et al. Prevalence of early dementia after first-ever stroke: a 24-year population-based study. Stroke. 2011;42(3):60712.

89. Ivan CS, Seshadri S, Beiser A, Au R, Kase CS, Kelly-Hayes M, et al. Dementia after stroke: the Framingham Study. Stroke. 2004;35(6):1264-8.

90. Sadowski M, Pankiewicz J, Scholtzova H, Li YS, Quartermain D, Duff K, et al. Links between the pathology of Alzheimer's disease and vascular dementia. Neurochem Res. 2004;29(6):1257-66

91. Newman SC. The prevalence of depression in Alzheimer's disease and vascular dementia in a population sample. J Affect Disord. 1999;52(1-3):169-76.

92. Kavirajan H, Schneider LS. Efficacy and adverse effects of cholinesterase inhibitors and memantine in vascular dementia: a meta-analysis of randomised controlled trials. Lancet Neurol. 2007;6(9):782-92.

93. Santos CO, Caeiro L, Ferro JM, Figueira ML. Mania and stroke: a systematic review. Cerebrovasc Dis. 2011:32(1):11-21.

94. Starkstein SE, Mayberg HS, Berthier ML, Fedoroff P, Price TR, Dannals RF, et al. Mania after brain injury: neuroradiological and metabolic findings. Ann Neurol. 1990;27(6):652-9.

95. Rabins PV, Starkstein SE, Robinson RG. Risk factors for developing atypical (schizophreniform) psychosis following stroke. J Neuropsychiatry Clin Neurosci. 1991;3(1):6-9.

96. Forstl H, Almeida OP, Owen AM, Burns A, Howard R. Psychiatric, neurological and medical aspects of misidentification syndromes: a review of 260 cases. Psychol Med. 1991;21(4):905-10.

97. Fleminger S, Burns A. The delusional misidentification syndromes in patients with and without evidence of organic cerebral disorder: a structured review of case reports. Biol Psychiatry. 1993;33(1):22-32

98. Feinberg TE, Shapiro RM. Misidentification: reduplication and the right hemisphere. Neuropsych Neuropsychol Behav Neurol. 1989;2(1):39-48.

99. Patterson MB, Mack JL. Neuropsychological analysis of a case of reduplicative paramnesia. J Clin Exp Neuropsychol. 1985;7(1):111-21.

100. Hudson AJ, Grace GM. Misidentification syndromes related to face specific area in the fusiform gyrus. J Neurol Neurosurg Psychiatry. 2000;69(5):645-8.

101. Signer SF. Localization and lateralization in the delusion of substitution. Capgras symptom and its variants. Psychopathology. 1994;27(3-5):168-76.

102. Ellis HD. The role of the right hemisphere in the Capgras delusion. Psychopathology 1994:27(3-5):177-85. 